\title{
Mining Experimental Data for Dynamical Invariants - From Cognitive Robotics to Computational Biology
}

\author{
Hod Lipson \\ Mechanical \& Aerospace Engineering and Computing \& Information Science \\ Cornell University \\ hod.lipson@cornell.edu
}

For centuries, scientists have attempted to identify and document analytical laws that underlie physical phenomena in nature. Despite the prevalence of computing power, the process of finding natural laws and their corresponding equations has resisted automation. A key challenge to finding analytic relations automatically is defining algorithmically what makes a correlation in observed data important and insightful. By seeking dynamical invariants, we go from finding just predictive models to finding deeper conservation laws. We demonstrated this approach by automatically searching motion-tracking data captured from various physical systems, ranging from simple harmonic oscillators to chaotic double-pendula. Without any prior knowledge about physics, kinematics, or geometry, the algorithm discovered Hamiltonians, Lagrangians, and other laws of geometric and momentum conservation. The discovery rate accelerated as laws found for simpler systems were used to bootstrap explanations for more complex systems, gradually uncovering the "alphabet" used to describe those systems. Applications to modeling physical and biological systems will be shown. 